\title{
Minimum QT Duration
}

National Cancer Institute

\section{Source}

National Cancer Institute. Minimum QT Duration. NCI Thesaurus. Code C62133.

The minimum duration (time) of the QT interval, obtained from a set of measurements of the QT interval. The QT interval is defined as the time from the beginning of the QRS complex to the end of the T wave, representing the time it takes for the ventricles to depolarize and subsequently repolarize. In some cases, the Q wave will be absent, in which case the QT interval is measured from the beg inning of the R wave to the end of the T wave. 